\title{
Prognostic Factors in Severe Falciparum Malaria
}

\author{
Polrat Wilairatana ${ }^{1,2^{*}}$, Noppadon Tangpukdee ${ }^{1,2}$ and Srivicha Krudsood ${ }^{1,3}$ \\ ${ }^{1}$ Malaria Clinical Research Unit, Malaria Excellence Center, Mahidol University, Thailand \\ ${ }^{2}$ Department of Clinical Tropical Medicine, Mahidol University, Thailand \\ ${ }^{3}$ Department of Tropical Hygiene, Mahidol University, Thailand
}

*Corresponding author: Polrat Wilairatana, Malaria Clinical Research Unit, Malaria Excellence Center and Department of Clinical Tropical Medicine, Faculty of Tropical Medicine, Mahidol University 420/6 Rajvithi Road, Rajthevi, Bangkok 10400, Thailand, Tel: 66-081-8602101; E-mail: polrat.wil@mahidol.ac.th

Received date: April 15, 2014; Accepted date: April 17, 2014; Published date: April 20, 2014

Copyright: (c) 2014 Wilairatana P. This is an open-access article distributed under the terms of the Creative Commons Attribution License, which permits unrestricted use, distribution, and reproduction in any medium, provided the original author and source are credited

\section{Editorial}

In 2012, prognostic factors in severe falciparum malaria were mentioned in World Health Organization (WHO)'s handbook of management of severe malaria [1]. Those factors included 9 clinical factors and 17 laboratory factors. Some factors reflect vital organ dysfunction similar to definition of severe falciparum malaria such as clinical signs of organ dysfunction (eg. renal injury, pulmonary edema), shock, respiratory distress (acidosis), hypoglycemia, hyperlactatemia, creatinine $>3 \mathrm{mg} / \mathrm{dl}$ with renal impairment and anemia with hemoglobin $<5 \mathrm{~g} / \mathrm{dl}$. Some factors indicate magnitude of disease severity such as deep coma, absent corneal reflexes, papilledema, hyperparasitemia $(>250,000 / \mu$ l or $>5 \%)$, blood urea nitrogen $>60 \mathrm{mg} / \mathrm{dl}$, high cerebrospinal (CSF) lactate $(>6 \mathrm{mmol} / \mathrm{l})$ and low CSF glucose, greater than 3 fold elevation in serum transaminases, increased plasma $5^{\prime}$-nucleotidase, raised muscle enzymes, low antitrombin III levels and very high plasma concentrations of tumor necrosis factor, and some factors indicate poor outcomes in severe malaria such as age $<3$ years, peripheral schizontemia, mature pigmented parasites $(>20 \%$ of parasites $)$, peripheral blood polymorphonuclear leukocytosis $(>12,000 / \mu \mathrm{l})$, mature pigmented parasites $(>20 \%$ of parasites $)$ and peripheral blood polymorphonuclear leukocytes with visible malaria pigment $(>5 \%)$. However these factors are not absolute and in some fatal cases many factors coexist [2]. Moreover some poor factors can be benign explanation such as hypoglycemia may occur in uncomplicated infections due to quinine-induced hyperinsulinemia [3]. The relation between parasitemia and prognosis varies according to malaria transmission level. In low-transmission areas, mortality from acute falciparum malaria begins to increase with parasite densities over $100,000 / \mu \mathrm{l}$ (around $2.5 \%$ parasitemia) whereas in higher transmission area much higher parasite densities may be well tolerated and the patients may present as benign disease [1]. Therefore the sensitivity and specificity of parasitemia alone as a prognostic factor is limited, but can be improved by staging parasites development (more mature parasites indicate worse prognosis), and looking at the number of polymorphonuclear leukocytes with visible malaria pigment $(>5 \%$ indicates poor prognosis) [3].
Tangpukdee et al. [4] showed that perpheral shizontemia was found in $39.6 \%$ of severe falciparum malaria patients. Shizontemia was also found in uncomplicated falciparum malaria turning to severe malaria even with artemisinin-combination therapy [5]. Shizontemia was shown to be an indicator of severe falciparum malaria and might be included in definition of severe falciparum malaria in the future.

Although WHO's handbook of management of severe malaria indicated only prognostic factors in severe falciparum malaria, these 'clinical' prognostic factors may be useful to severe malaria patients from non-falciparum species. However, the 'laboratory' prognostic factors for severe malaria patients from non-falciparum species should be further studied for recommendation of prognostic factors in the future malaria treatment guidelines.

\section{Acknowledgements}

The authors thank the Office of the Higher Education Commission and Mahidol University under the National Research Universities Initiative; and also the Faculty of Tropical Medicine, Mahidol University, Thailand for kind support.

\section{References}

1. WHO (2012) Management of severe malaria (3rd edn). WHO, Geneva.

2. WHO (2010) Guidelines for the treatment of malaria. Second edition. WHO, Geneva.

3. Cook GC, Zumla AI (2009) Manson's Tropical diseases, 22nd Edition. Saunders-Elsevier, China.

4. Tangpukdee N, Krudsood S, Wilairatana P (2013) Schizontemia as an indicator of severe malaria. Southeast Asian J Trop Med Public Health 44: 740-743.

5. Tangpukdee N, Krudsood S, Thanachartwet V, Duangdee C, Paksala S, et al. (2007) Predictive score of uncomplicated falciparum malaria patients turning to severe malaria. Korean J Parasitol 45: 273-282. 